\title{
SCALAR DEPENDENT ALGEBRAS
}

\author{
RAYMOND COUGHLIN, ERWIN KLEINFELD AND MICHAEL RICH ${ }^{1}$
}

\begin{abstract}
An algebra $A$ over a field $F$ will be called scalar dependent in case for every $x, y, z$ in $A$ there exists a function $g(x, y, z)$ in $F$ such that $(x y) z=g(x, y, z) x(y z)$. The main result of this paper is that any scalar dependent algebra which contains a nonzero idempotent must always be associative. Since there are known to exist scalar dependent algebras which are not associative, the hypothesis regarding the existence of an idempotent is actually necessary.
\end{abstract}

In an earlier study of scalar dependent algebras [1], two of the authors showed that third-power-associative scalar dependent algebras which contain an identity element 1 and a nonzero idempotent $e \neq 1$ must be associative. Here we shall prove the same result with considerably weaker assumptions. Throughout $A$ will denote a scalar dependent algebra over $F$. If $x, y, z$ are elements of $A$ then the associator $(x, y, z)$ is defined by $(x, y, z)=(x y) z-x(y z)$. Essential to many of our calculations is the fact that $(x, y, z)=(\alpha-1) x(y z)$ for some element $\alpha$ in $F$.

THEOREM 1. Every scalar dependent algebra with identity element 1 must be associative.

Proof. We note that in order for the theorem to be false there must be elements $x, y, z$ in $A$ such that $(x, y, z) \neq 0$. But then $(\alpha-1) x(y z)=$ $(x, y, z)=(x+1, y, z)=(\beta-1)(x+1)(y z)$. Solving this equation we have

$$
(\alpha-\beta) x(y z)=(\beta-1) y z \text {. }
$$

If $\alpha=\beta$, then it follows from (1) that $(\beta-1) y z=0$, so that either $\beta=1$, or $y z=0$. Either of these are sufficient to imply $(x, y, z)=0$, contrary to assumption. Thus $\alpha \neq \beta$. But then (1) implies that $x(y z)$ is a scalar multiple of $y z$. Since $(x, y, z)$ is a scalar multiple of $x(y z)$, then

$$
(x, y, z)=\gamma(y z) \text {, for some } \gamma \text { in } F \text {. }
$$

Received by the editors August 2, 1972.

AMS (MOS) subject classifications (1970). Primary 17A30, 17E05.

Key words and phrases. Associator, scalar dependent algebra.

${ }^{1}$ The research of the first author was supported in part by a Temple University Summer Research Grant. The research of the second author was supported in part by the National Science Foundation under GP-32898X.

(c) American Mathematical Society 1973 
In (2) we may replace $z$ by $z+1$, since $(x, y, z+1)=(x, y, z) \neq 0$, thus obtaining

$$
(x, y, z+1)=\gamma^{\prime}(y)(z+1), \text { for some } \gamma^{\prime} \text { in } F .
$$

Solving (2) and (3) simultaneously, we get

$$
\left(\gamma-\gamma^{\prime}\right) y z=\gamma^{\prime} y \text {. }
$$

If $\gamma^{\prime}=\gamma$, then (4) implies $\gamma^{\prime} y=0$, so that either $\gamma^{\prime}=0$, or $y=0$. Since both of these imply $(x, y, z)=0$, contrary to assumption, we must have $\gamma^{\prime} \neq \gamma$. But then (4) may be solved for $y z$ as a scalar multiple of $y$. This combined with (2) leads to

$$
(x, y, z)=\delta y, \text { for some } \delta \text { in } F .
$$

Replacing $y$ by $y+1$ in (5) one gets

$$
(x, y+1, z)=\delta^{\prime}(y+1), \text { for some } \delta^{\prime} \text { in } F .
$$

Solving (5) and (6) simultaneously implies

$$
\left(\delta-\delta^{\prime}\right) y=\delta^{\prime} .
$$

If $\delta^{\prime}=\delta$ then (7) implies $\delta^{\prime}=0$, so that $(x, y, z)=0$, using (6). Since $(x, y, z) \neq 0, \delta^{\prime} \neq \delta$. But then (7) implies that $y$ is a scalar multiple of 1 , implying $(x, y, z)=0$. By assuming $(x, y, z) \neq 0$, we have reached a contradiction. Consequently $A$ must be associative. This completes the proof of the theorem.

Lemma 1. Let $e$ be an idempotent in $A$. Then $(e, e, A)=(e, A, e)=$ $(A, e, e)=0$.

Proof. Let $x$ be an arbitrary element of $A$. We show first that $(x, e, e)=$ 0 . Note that $(x, e, e)=(\alpha-1) x e$. But then $(x, e, e)=(x+e, e, e)=$ $(\beta-1)(x+e) e$. Solving we get $(\alpha-\beta) x e=(\beta-1) e$. If $\alpha=\beta$ then $(\beta-1) e=0$, so that either $\beta=1$, or $e=0$. In both instances $(x, e, e)=0$, and we are done. Thus $\alpha \neq \beta$, so that $x e$ is a scalar multiple of $e$. If $x e=\gamma e$, then $(x, e, e)=(x e) e-x e=\gamma e-\gamma e=0$. This shows $(x, e, e)=0$, in all cases. Next we show $(e, e, x)=0$. We see that $(e, e, x)=(\alpha-1) e(e x)=(e, e, e+x)$ $=(\beta-1) e(e+e x)$. Solving we get $(\alpha-\beta) e(e x)=(\beta-1) e$. If $\alpha=\beta$ then either $\beta=1$, or $e=0$. In both instances $(e, e, x)=0$, so that we can assume $\alpha \neq \beta$. But then $e(e x)$ must be a scalar multiple of $e$. Since $e x=e^{2} x=\alpha e(e x)$, then $e x$ must also be a scalar multiple of $e$, say $e x=\delta e$. Thus $(e, e, x)=$ $e x-e(e x)=\delta e-\delta e=0$. Next we show $(e, x, e) e=0$. Let $\rho=g(e, x-e x, e)$. Then using $(e, e, x)=0=(x, e, e)$, we have $(e, x, e) e=[(e x) e-e(x e)] e=$ $(e x) e-[e(x e)] e=[e x-e(x e)] e=[e(x-x e)] e=\rho e[(x-x e) e]=\rho e[x e-(x e) e]$ $=-\rho e(x, e, e)=0$. Since $(e, x, e)=(\alpha-1) e(x e)=(e, x+e, e)=(\beta-1) \times$ $e(x e+e)$, we have $(\alpha-\beta) e(x e)=(\beta-1) e$. If $\alpha=\beta$, then $(\beta-1) e=0$, so 
that either $\beta=1$, or $e=0$, and we are done. Assume that $\alpha \neq \beta$. Then $e(x e)$ is a scalar multiple of $e$, say $e(x e)=\gamma e$. Then $(e, x, e)=(\alpha-1) \gamma e=$ $\rho e$. Since $(e, x, e) e=0$, we get $(\rho e) e=0=\rho e=(e, x, e)$, and we are done. This completes the proof of the lemma. It is well known that Lemma 1 implies that $A$ has a Peirce decomposition as a direct sum $A=A_{11} \oplus A_{10} \oplus$ $A_{01} \oplus A_{00}$, where $x_{i j} \in A_{i j}, i, j=0,1$ if and only if $e\left(x_{i j}\right)=i x_{i j},\left(x_{i j}\right) e=j x_{i j}$, relative to any idempotent $e$.

Lemma 2. Suppose $e$ is an idempotent and $x, y$ arbitrary elements in $A$. If $(x, y, e) \neq 0$, then either there exist $\alpha, \beta$ in $F$ such that $(x, y, e)=\alpha x e=$ $\beta e$, or $(x, y, e)=\beta e$ and $x e=0$. Similarly if $(x, e, y) \neq 0$, then $(x, e, y)=$ $\gamma e y=\delta e$. Finally if $(e, x, y) \neq 0$, then either $(e, x, y)=\rho e y=\sigma e$, or $(e, x, y)=$ $\sigma e$ and $e y=0$.

Proof. We have $(x, y, e)=(\alpha-1) x(y e)$. Substituting $y+e$ for $y$, we have $(x, y, e)=(x, y+e, e)=(\beta-1) x(y e+e)$, so that $(\alpha-\beta) x(y e)=$ $(\beta-1) x e$. If $\alpha \neq \beta$, then $x(y e)$ is a scalar multiple of $x e$, and hence so is $(x, y, e)$. If on the other hand $\alpha=\beta$, then $(\beta-1) x e=0$, so that either $\beta=1$, or $x e=0$. We have shown that either $(x, y, e)$ is a scalar multiple of $x e$, or $x e=0$. In this last assertion replace $x$ by $x+e$. Then Lemma 1 implies $(x+e, y, e)=(x, y, e) \neq 0$. Thus either $(x, y, e)$ is a scalar multiple of $x e+e$ or $x e+e=0$. If we consider each of the four possibilities separately the first part of the lemma becomes obvious. The other two parts are similar and are left to the reader, noting that if $e y=0$, then $(x, e, y)=0$.

Definition. The right nucleus $R$ of $A$ is defined as

$$
R=\{a \in A \mid(A, A, a)=0\} .
$$

The nucleus is the intersection of right, middle and left nucleus.

LEMmA 3. Every idempotent e belongs to the right nucleus $R$.

Proof. Assume $e$ is not in $R$. Then there exist elements $x, y$ in $A$ such that $(x, y, e) \neq 0$. Using Lemma 2 , there exists $\beta$ in $F$ such that $(x, y, e)=\beta e$. By definition we have $(x, y e, e)+(x, y, e) e=[x(y e)] e-$ $x[(y e) e]+[(x y) e] e-[x(y e)] e=(x y) e-x(y e)=(x, y, e)$. But then $(x, y e, e)$ $+\beta e=\beta e$, so that $(x, y e, e)=0$. Thus $\left(x, y_{11}+y_{01}, e\right)=0$. But if $y e=0$, then $(x, y, e)=(\alpha-1) x(y e)=0$, so that $\left(x, y_{00}+y_{10}, e\right)=0$. We have proved $(x, y, e)=0$, which is contrary to assumption. Thus $e$ must be in $R$. This completes the proof of the lemma.

Lemma 4. $A_{10}$ and $A_{01}$ are contained in the right nucleus $R$.

Proof. We have $x_{01} y_{01}=\left(x_{01} e\right) y_{01}=(\gamma-1) x_{01}\left(e y_{01}\right)=0$. This implies $\left(e+x_{01}\right)^{2}=e+x_{01}$, by expansion. But then Lemma 3 implies that $e+x_{01}$ 
belongs to $R$. Since $R$ is a subring of $A$, we have $x_{01}$ in $R$. Then $\left(x_{10}, e, y_{10}\right)=$ $-x_{10} y_{10}=\delta e$, using Lemma 2. But Lemma 3 implies $0=\left(x_{10}, y_{10}, e\right)=$ $\left(x_{10} y_{10}\right) e=-(\delta e) e=-\delta e=x_{10} y_{10}$. Thus $\left(e+x_{10}\right)^{2}=e+x_{10}$. As before then $e+x_{10}$ is in $R$, hence $x_{10}$ is. This completes the proof of the lemma.

We are now ready to prove our main result.

THEOREM 2. If A contains a nonzero idempotent e, then $A$ is associative.

ProOf. Since $e$ belongs to $R$, we get through expansion that $(x, y, z e)=$ $(x y)(z e)-x[y(z e)]=[(x y) z] e-x[(y z) e]=[(x y) z] e-[x(y z)] e=(x, y, z) e$, for arbitrary $x, y, z$ in $A$. But then Lemma 2 implies $\left(x, e, y_{00}\right)=\beta e=(\beta e) e=$ $\left(x, e, y_{00}\right) e=\left(x, e, y_{00} e\right)=0$. Similarly $\left(e, x, y_{00}\right)=\beta^{\prime} e=\left(\beta^{\prime} e\right) e=\left(e, x, y_{00}\right) e$ $=\left(e, x, y_{00} e\right)=0$. Using Lemma 2 , since $e y_{11}=y_{11} \neq 0$, we have $\left(x, e, y_{11}\right)=$ $\gamma e y_{11}=\delta e$. If $\gamma=0$, then $\left(x, e, y_{11}\right)=0$. If not then $y_{11}=\rho e$ implies $\left(x, e, y_{11}\right)=0$. Thus in either case we wind up with $\left(x, e, y_{11}\right)=0$. Then $\left(e, x, y_{11}\right)=\sigma e y_{11}=\tau e$ because of Lemma 2. Hence either $\sigma=0$, or $y_{11}=$ $\omega e$. The latter implies $\left(e, x, y_{11}\right)=0$, because of Lemma 1 , as of course the first possibility does too. Now Lemma 4 suffices to prove that $e$ belongs to the nucleus $N$ of $A$. From this it follows at once that $A_{i j} A_{k l} \subset \delta_{j k} A_{i l}$. Also since $e+x_{10}$ and $e+x_{01}$ belong to $N$ we must have $A_{10}+A_{01} \subset N$. In fact the only associators which are not known to be zero at this point are $\left(x_{11}, y_{11}, z_{11}\right)$ and $\left(x_{00}, y_{00}, z_{00}\right)$. However $e$ is the identity element of $A_{11}$ and so Theorem 1 implies that $\left(x_{11}, y_{11}, z_{11}\right)=0$. Next $\left(e+x_{00}, e+y_{00}\right.$, $\left.e+z_{00}\right)=\left(x_{00}, y_{00}, z_{00}\right)=(\alpha-1) x_{00}\left(y_{00} z_{00}\right)=(\beta-1)\left[e+x_{00}\right]\left[\left(e+y_{00}\right)\left(e+z_{00}\right)\right]$ $=(\beta-1) e+(\beta-1) x_{00}\left(y_{00} z_{00}\right)$. Thus $(\alpha-\beta) x_{00}\left(y_{00} z_{00}\right)=(\beta-1) e$ must be an element in the intersection of $A_{00}$ and $A_{11}$, and hence zero. Thus $\beta=1$, and so $\left(x_{00}, y_{00}, z_{00}\right)=0$. This completes the proof of the theorem.

\section{BIBLIOGRAPHY}

1. R. Coughlin and M. Rich, On scalar dependent algebras, Canad. J. Math. 24 (1972), 696-702.

Department of Mathematics, Temple University, Philadelphia, Pennsylvania 19122

Department of Mathematics, University of Iowa, Iowa City, Iowa 52240 